\title{
Molecular

\section{Dynamics of Srf, p300 and histone modifications during cardiac maturation in mouse $\dagger$}

\author{
Markus Schueler, $\ddagger^{a b c}$ Qin Zhang, $\ddagger^{a d}$ Jenny Schlesinger, ${ }^{a c e}$ Martje Tönjes ${ }^{a}$ and \\ Silke R. Sperling*ac
}

Received 7th September 2011, Accepted 27th October 2011

DOI: $10.1039 / \mathrm{c} 1 \mathrm{mb05363a}$

The adaptation of the cellular network to functional changes, timing and patterning of gene expression is regulated by binding of transcription factors to gene regulatory elements, which in turn depends on co-occurring histone modifications. These two layers influence each other, enabling a further level of regulatory fine-tuning. We analyzed the interdependencies between histone 3 acetylation, histone 3 lysine 4 dimethylation, the transcription factor Srf and the histone acetyltransferase p300 in an in vivo model using chromatin immunoprecipitation in a time-series during cardiac maturation in mouse. We found a strong correlation between the presence of the two histone modifications and binding of Srf and p300. Using linear modeling techniques we could show that each factor contributes individually as well as conjointly to histone 3 acetylation and gene expression, probably aided by accompanying histone 3 lysine 4 dimethylation. We further demonstrate that changes in gene expression during cardiac maturation are attended by changes of the analyzed regulators while revealing a high variability of combinatorial regulation. Finally, we propose a model of Srf-driven gene expression in cardiomyocytes.

\section{Introduction}

Transcription factors can regulate the expression of genes in a tissue-specific and quantitative manner and are thus major regulators of embryonic developmental processes. The ability of transcription factors to bind to DNA is highly dependent on the accessibility of their binding sites. The majority of genomic DNA in eukaryotes is packaged into chromatin by association with histone proteins. Covalent modifications of histone tails and ATP-dependent chromatin remodeling facilitate access for DNA-binding transcription factors. ${ }^{1}$ Thus, to understand networks directing gene expression the interplay between transcription factors, co-regulatory elements and epigenetic factors has to be considered.

${ }^{a}$ Cardiovascular Genetics Group, Department of Vertebrate Genomics, Max Planck Institute for Molecular Genetics, Berlin, Germany.

E-mail: silke.sperling@charite.de

${ }^{b}$ Department of Computational Molecular Biology,

Max Planck Institute for Molecular Genetics, Berlin, Germany

${ }^{c}$ Experimental and Clinical Research Center, Charité - University

Medicine Berlin and Max Delbrück Center for Molecular Medicine,

Berlin, Germany

${ }^{d}$ Shaanxi Key Laboratory of Molecular Biology for Agriculture,

College of Animal Science and Technology.

Northwest A\& F University, Yangling, China

${ }^{e}$ Department of Biology, Chemistry and Pharmacy,

Free University Berlin, Berlin, Germany

$\dagger$ Electronic supplementary information (ESI) available. See DOI:

$10.1039 / \mathrm{clmb} 05363 \mathrm{a}$

$\ddagger$ These authors contributed equally to this work.
Serum Response Factor (Srf) is an ubiquitously expressed MADS (MCM1, Agamous, Deficiens, SRF) box transcription factor $^{2}$ and binds to a DNA consensus sequence known as the CArG box $\left[\mathrm{CC}(\mathrm{A} / \mathrm{T})_{6} \mathrm{GG}\right]$. Despite the ubiquitous expression of SRF, the CArG box is predominantly found in promoters of muscle- and growth-factor-associated genes. ${ }^{3,4}$ Functional analyses showed that embryonic stem cells lacking Srf display defects in spreading, adhesion and migration. These defects correlate with defective formation of cytoskeletal structures. ${ }^{5}$ In Srf-null neonatal cardiomyocytes, severe defects in the contractile apparatus are observed. ${ }^{6}$ Srf-deficient mice embryos $\left(\mathrm{Srf}^{-1-}\right)$ show a gastrulation defect, fail to develop the mesoderm and subsequently die between E8.5 and E12.5. ${ }^{7}$ Cre mice with knockout of Srf in $80 \%$ of the cardiomyocytes display severe heart defects and die at E11.5 and Cre mice lacking skeletal muscle Srf expression die during the perinatal period from severe skeletal muscle hypoplasia. ${ }^{9}$

Studies analysing co-occurring histone modifications showed that histone $\mathrm{H} 3$ and $\mathrm{H} 4$ acetylation ( $\mathrm{H} 3 \mathrm{ac}$ and $\mathrm{H} 4 \mathrm{ac}$ ) are enriched at $\mathrm{CArG}$ boxes in cultured smooth muscle cells (SMCs). ${ }^{3,10-12}$ Additionally, SMC genes show higher levels of H3 Lys4 dimethylation (H3K4me2) relative to non-SMC lineages. ${ }^{3}$ A model of step-wise molecular events directing the activation of SMC gene transcription has been depicted by McDonalds and Owens ${ }^{13}$ (Fig. 1A). It comprises the combinatorial enrichment of both $\mathrm{H} 3 \mathrm{~K} 4 \mathrm{me} 2$ and $\mathrm{H} 4 \mathrm{ac}$ at regulatory CArG boxes of SMC genes. $\mathrm{H} 4 \mathrm{ac}$ is thought to lead to an open chromatin state facilitating genomic access while $\mathrm{H} 3 \mathrm{~K} 4 \mathrm{me} 2$ provides a docking site to the 
A
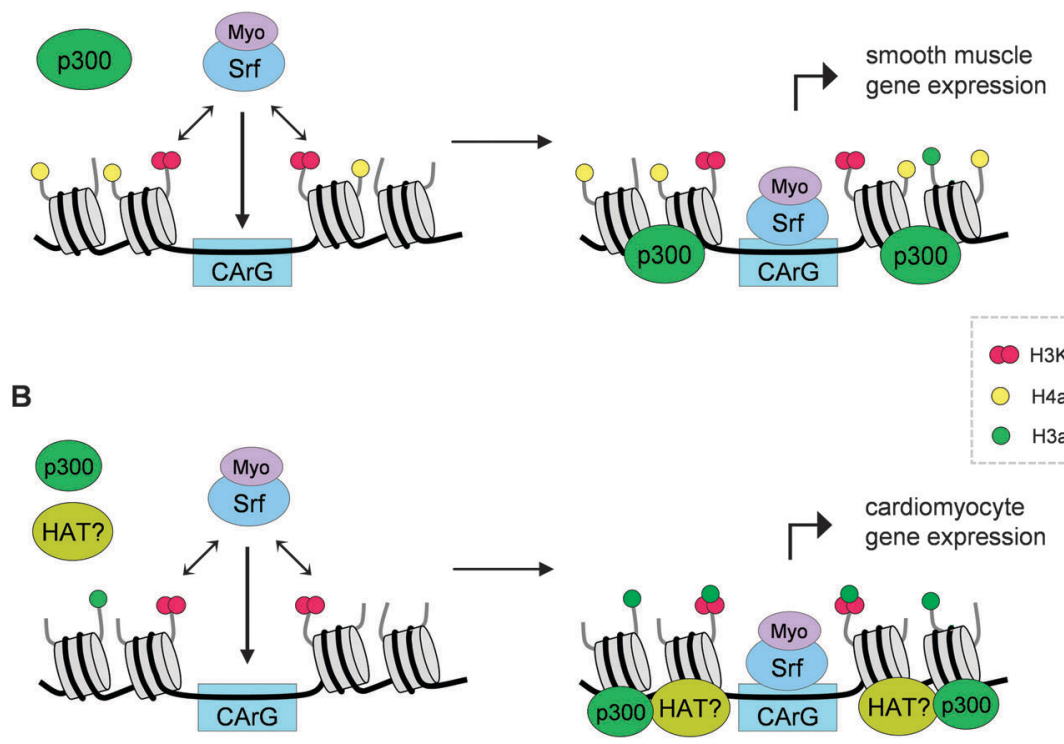

Fig. 1 (A) Model for smooth muscle cell (SMC) gene activation adapted from McDonald and Owens. ${ }^{13}$ H4ac and H3K4me2 facilitate the binding of the Srf/Myocardin complex to regulatory CArG boxes. The complex then recruits p300, leading to further histone acetylation and active gene expression. (B) Model for Srf-driven gene expression in cardiomyocytes. In contrast to SMC, H3ac takes over the role of H4ac in facilitating gene expression. Furthermore, histone acetylation by p300 is accompanied by additional so far unexplored histone acetyltransferases (HAT).

Srf/Myocardin complex. ${ }^{13}$ Myocardin, one of the most potent co-activators of Srf, belongs to the SAP domain family of nuclear proteins and is exclusively expressed in SMCs and cardiomyocytes. ${ }^{14}$ Binding of the Srf/Myocardin complex to the CArG box promotes the recruitment of other transcription factors via the powerful C-term transcriptional activation domain of Myocardin. Thus, the recruitment of the acetyltransferase p300 establishes additional histone acetylation marks which in turn activate transcription. ${ }^{13}$ p300 is a known histone acetyltransferase and transcriptional co-activator, which was found to reside in enhancer and promoter regions. ${ }^{15,16}$

The model proposed has been established based on studies in SMCs, however, a systematic validation in an in vivo model comprising the dynamics of the interregulatory dependencies is pending. Further, the relevance of factor interaction for gene transcription in cardiomyocytes is unknown. Recently, we showed that the transcriptional activity of Srf in the mouse cardiomyocyte cell line HL-1 is highly depending on the co-occurrence of H3ac. In contrast to SMCs H4ac marks do not enhance Srf-driven gene expression in cardiomyocytes. ${ }^{17}$ To gain further insights, we used a time-series approach of mouse hearts and monitored the combinatorial regulation of gene expression driven by Srf, H3ac, H3K4me2, and p300. The dynamic changes of transcription factor binding and depletion or enrichment of histone modifications were studied during the cardiac maturation process occurring at the prenatal and postnatal developmental phase (E18.5, P0.5 and P4.5). Based on our previous genome-wide study ${ }^{17}$ we selected 190 cardiac enhancer and promoter regions for this focused approach and used chromatin immunoprecipitation (ChIP) followed by highthroughput qPCR to quantify respective dynamics. In addition, we measured the corresponding changes of genes expression showing the functional consequences of the observed alterations of factor binding and histone modifications. Based on our findings we propose a model of Srf-driven gene expression in cardiomyocytes (Fig. 1B).

\section{Results and discussion}

Our previous analysis performed in a cardiomyocyte cell line (HL-1) showed that the activating potential of Srf is significantly depending on accompanying H3ac. ${ }^{17}$ In contrast to the Srf-driven gene expression model proposed for SMC, we do not observe a dependency for $\mathrm{H} 4 \mathrm{ac}$ or $\mathrm{H} 3 \mathrm{~K} 4 \mathrm{me} 2$ (Fig. 2). To further elaborate on the dynamic relationship between Srf and histone modifications, we switched towards an in vivo timeseries model of cardiac maturation in mouse and investigated

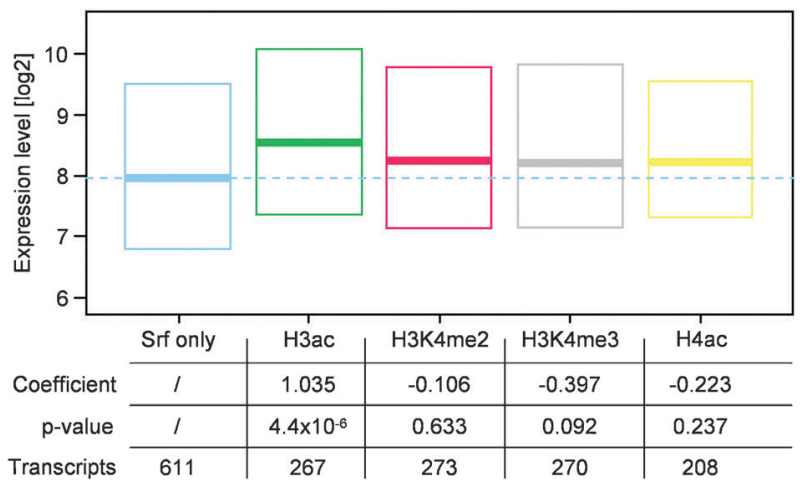

Fig. 2 The influence of histone modifications at Srf binding sites on expression levels. Boxplots for the expression of transcripts with $\mathrm{Srf}$ binding site and specific histone modifications compared to genes with Srf binding site but no histone modification. A single transcript can belong to multiple groups if it has multiple histone modifications. Estimates for the influence of individual histone modifications and their associated $p$-values are derived by an ANOVA model. Figure taken from Schlesinger et al., ${ }^{17}$ modified. 
A

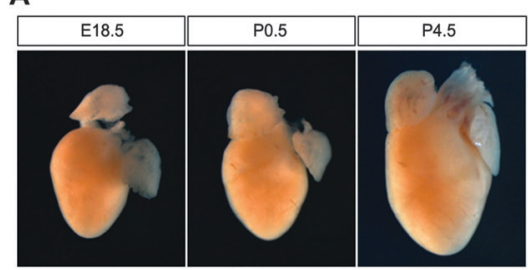

B
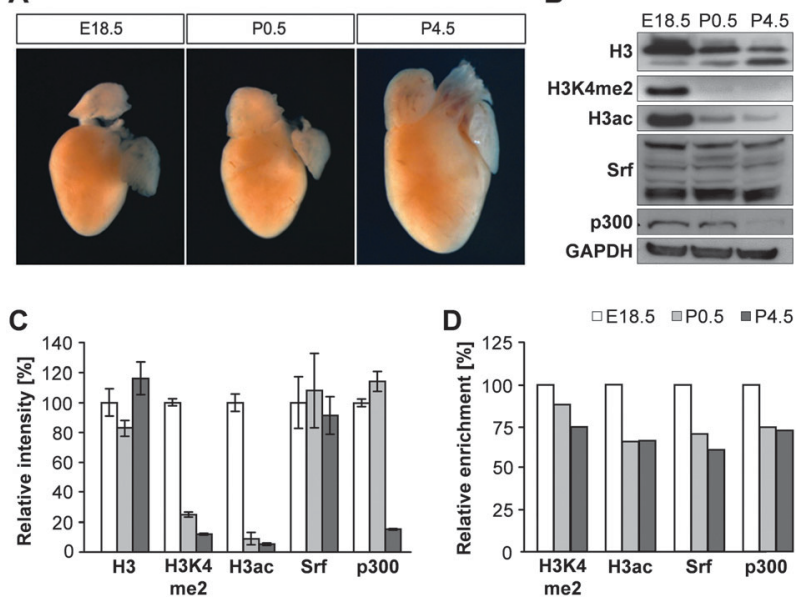

Fig. 3 (A) Three stages of mouse hearts during cardiomyocyte maturation around birth. Figure taken from Schlesinger et al., ${ }^{17}$ modified. (B) Western Blot analysis of protein expression in mouse hearts for Srf, p300, H3ac, H3K4me2 and H3. GAPDH served as loading control. Srf is expressed in four different isoforms. The H3 level is divided in phosphorylated H3 (upper band) and non-phosphorylated H3 (lower band). (C) Barplot of relative protein expressions of Srf, p300 and H3 and relative levels of histone modifications $\mathrm{H} 3 \mathrm{ac}$ and $\mathrm{H} 3 \mathrm{~K} 4 \mathrm{me} 2$. Total protein amounts were gathered using repeated densitometry measurements. All intensities are normalized to GAPDH and shown relative to E18.5. (D) Barplot of relative average ChIP level over all regions for every factor in every stage. Measurements after $\Delta \mathrm{CP}$ normalization showing a distinct trend between the individual stages. Consequently, an additional linear shift was introduced to remove the trend.

the relationship between binding of Srf, the acetyltransferase p300 and $\mathrm{H} 3 \mathrm{ac}$ as well as $\mathrm{H} 3 \mathrm{~K} 4 \mathrm{me} 2$. We included $\mathrm{H} 3 \mathrm{~K} 4 \mathrm{me} 2$ in our further studies even though we did not observe an enhancement of Srf-driven gene expression in our cell line analyses. H3K4me2 had been shown to function as a stabilizing element for Srf binding and therefore might act in concert with H3ac. This could potentially be underlined by our previous observation that co-localization of $\mathrm{H} 3 \mathrm{ac}$ and $\mathrm{H} 3 \mathrm{~K} 4 \mathrm{me} 2$ is associated with higher gene expression than isolated H3K4me 2 marks. ${ }^{18}$

We used mouse hearts of three developmental stages (Fig. 3A), one prenatal stage (E18.5) and two postnatal stages (P0.5, P4.5). From the fetal to the postnatal stage, the heart adapts to the body circulation and cardiomyocytes mature. During this process the heart increases in size, the cells elongate, myofibrils align and cell-cell contacts become bipolar. ${ }^{19}$

As an initial step, we determined total protein levels of Srf, p300, H3ac, H3K4me2, total H3 and GAPDH (normalization control) at all stages using Western Blot followed by densitometric measurements of the resulting bands (Fig. 3B and C). Due to alternative splicing events of Srf, four different isoforms are expressed in the heart, ${ }^{20,21}$ showing only slight changes during the time-series. The level of p300 is strongly reduced at $\mathrm{P} 4.5$ (85\% compared to E18.5). Both, the levels of $\mathrm{H} 3 \mathrm{ac}$ and $\mathrm{H} 3 \mathrm{~K} 4 \mathrm{me} 2$, show a high reduction directly after birth (75-91\% compared to E18.5) whereas the total amount of $\mathrm{H} 3$ remains nearly constant.

We selected enhancer and promoter regions with potential Srf-driven regulatory influence on gene expression based on our previous genome-wide ChIP-chip/seq data of histone modifications and Srf binding (see Fischer et al. ${ }^{18}$ and Schlesinger et al. ${ }^{17}$ ). A priori 422 genomic regions that shared enrichment for at least two of $\mathrm{H} 3 \mathrm{ac}, \mathrm{H} 3 \mathrm{~K} 4 \mathrm{me} 2$ and Srf binding were selected located in a $10 \mathrm{~kb}$ window of genes involved in cardiac development. We used a multistep filtering approach to focus on genes with either heart or muscle annotation as a GO term, an entry of cardiac disease in OMIM or relevance in a manually curated literature search. Genomic regions of a maximum of $500 \mathrm{bp}$ in length were defined (Fig. S1, ESI $\dagger$ ) and Taq Man assays for the following ChIP analysis were established for every window. Windows without unique primer pairs were discarded from the analysis. This resulted in a total of 190 genomic regions in the proximity of heart and muscle developmental genes, which were consequently analyzed.

ChIP followed by real-time qPCR was performed for each factor plus input DNA for each region with samples of mouse hearts of the time points E18.5, P0.5 and P4.5 in triplicates. This resulted in a total of 7578 measurements, which fulfilled our quality standards. Data normalization was performed computing $\triangle \mathrm{CP}$ values using the measured input DNA. As a proof of principle, the resulting ChIP enrichments from stage P0.5 were compared to our previous ChIP-chip/seq results in HL-1 cells (Fig. S2, ESI $\dagger$ ), which confirmed the reliability of the system. As we were interested in the dynamic changes of factor binding and histone modification over the time course, we checked for a potential overall bias, which might be introduced by the observed changes of the protein content during the time course. Consequently, we compared the average ChIP enrichment over all regions for each factor in each stage and found differences between the three stages (Fig. 3D and Fig. S3A, $\mathrm{ESI} \dagger)$ recapitulating the findings from the overall protein analysis (Fig. 3B and C). We eliminated this bias, as it would result in a general trend between individual stages and superficially high correlations between the individual factors. Therefore, we introduced a fixed linear shift for each single region and each single factor resulting in the same average enrichment in each stage for each factor (Fig. S3B, ESI $\dagger$ ). Finally, we calculated for each factor fold changes of ChIP enrichments between consecutive stages and grouped all changes into the categories significantly up, significantly down or unchanged according to a $t$-test using a significance level of $95 \%$.

As a first analysis, scatter plots were used to visualize the level of correlation between the fold changes of individual factors (Fig. 4). We found high to modest correlation for all the measured factors, with Pearson correlation coefficients ranging from 0.753 to 0.364 , which implicates that all factors are related to each other. Statistical significance of found correlation coefficients was evaluated using random experiments. As a null model, triplicate measurements for one factor and time point were randomly assigned to all regions without replacement and fold changes were again computed between successive timepoints. This process was repeated 20000 times and an empirical $p$-value was derived by counting the number of tries where the random correlation coefficient exceeded the true coefficient. Applying a significance level of $99.9 \%$ we found significant correlation for all of the analyzed pairings (Fig. S4, ESI $\dagger$ ). 
A

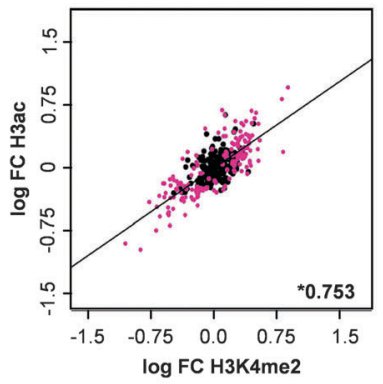

D

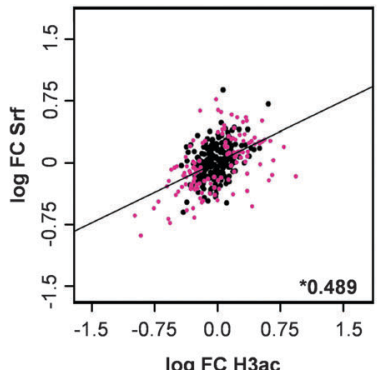

B

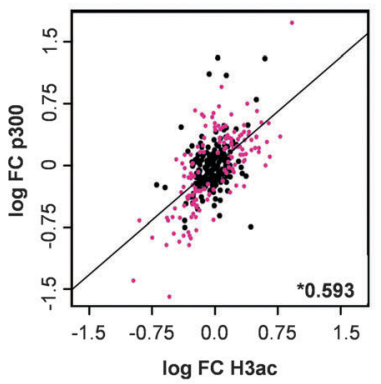

E

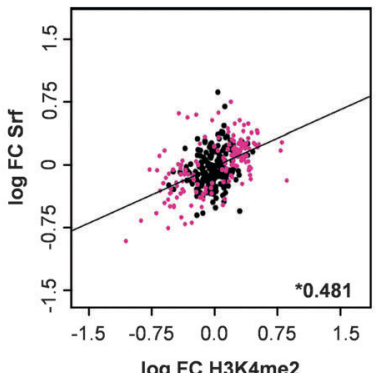

C

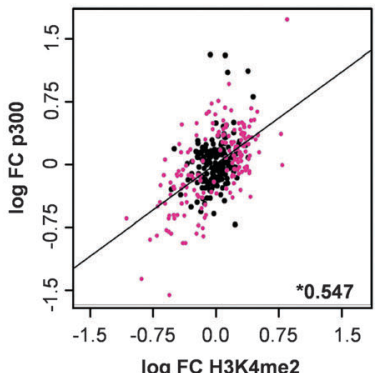

$\mathbf{F}$

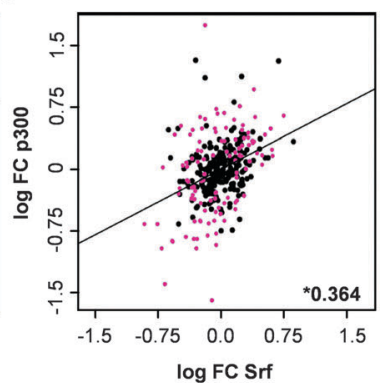

Fig. 4 Scatter plots of fold changes between the measured factors (combined time points). Red dots represent measurements with significant change for at least one of the factors. Lines represent the best linear fit for all measurements. Pearson correlation coefficients for all measurements are indicated in the lower right corner. Significance using an empirical $p$-value $<0.001$ is indicated by *.

The highest correlation coefficient of 0.753 was found between the changes in $\mathrm{H} 3 \mathrm{ac}$ and $\mathrm{H} 3 \mathrm{~K} 4 \mathrm{me} 2$ which is in accordance to the high correlation of absolute $\mathrm{H} 3 \mathrm{ac}$ and $\mathrm{H} 3 \mathrm{~K} 4 \mathrm{me} 2$ levels found by us ${ }^{17}$ and others. ${ }^{22} \mathrm{~A}$ recent study performed by Wang et al. ${ }^{23}$ suggested that $\mathrm{H} 3 \mathrm{~K} 4$ methylation might directly facilitate histone acetylation events. The second highest correlation coefficient (0.593) was found between changes in H3ac enrichment and p300 followed by p300 and H3K4me2 (0.547). While the correlation between changes in $\mathrm{p} 300$ and acetylation level was highly expected given the function of $\mathrm{p} 300$, the correlation between p300 and methylation level is of high interest. A possible explanation for this correlation supposed by Pray-Grant et al. ${ }^{24}$ and Wysocka et al. ${ }^{25}$ is an initial opening of chromatin through recruitment of ATP-dependent chromatin remodeling complexes induced by histone methylation which in turn allows p300 to bind. Another possibility might be the direct recognition of methylated sites by histone acetylation complexes (including p300) as suggested by Martin et $a l .{ }^{26}$ In addition, significant correlation was found between Srf and H3ac (0.489) as well as Srf and H3K4me2 (0.481) supporting the model by McDonald and Owens ${ }^{13}$ which suggest stabilization of Srf binding to the CArG box DNA motif via Myocardin or some Myocardin related factors that directly bind methylated histones. Further, this finding is in line with our previous observation in HL-1 cells such that siRNA mediated knockdown of Srf leads to a significant reduction of $\mathrm{H} 3$ acetylation at related genomic sites. ${ }^{17}$ Consistent with the model in SMCs, significant correlation was found between changes in Srf and p300. However, given that the observed correlation (0.364) between these two factors is much weaker than between any of the other measured factors and especially between Srf and H3ac might suggest additional mechanisms of Srf-triggered acetylation, which are independent from $\mathrm{p} 300$ binding.
While our first analysis of correlation between changes in the individual factors was based on the combination of fold changes between all three stages, we also considered each fold change individually. In general, fold changes as well as correlation coefficients were higher for changes between E18.5 and P0.5 than between P0.5 and P4.5 while, except for Srf and p300, still remaining significant. A possible explanation is that the physiological and cellular change reflected in the molecular setting is greater from the pre- towards the postnatal phase than later on. This might cause a higher influence of experimental noise on the correlation.

To analyze the interdependency in regulation of the measured factors, we used ANOVA linear models to predict changes in H3ac modification levels from changes in binding levels of $\mathrm{p} 300$ and Srf. We excluded the data for $\mathrm{H} 3 \mathrm{~K} 4 \mathrm{me}$ changes as these were shown to be highly correlated with the dependent variable $\mathrm{H} 3 \mathrm{ac}$ and could therefore obscure the models. After estimating coefficients for each of the predictive groups using a least-square approach, it was tested whether any of the coefficients was significantly different from zero implying a significant influence on changes in H3ac level. As before, randomized experiments were used to derive empirical $p$-values for the individual models, now using the percentage of variance explained or $R^{2}$ as a measurement for the goodness of fit for each model. ${ }^{27}$ The empirical $p$-value is therefore determined by the number of random models which yielded a higher $R^{2}$ when compared to the original model. Two single-factor ANOVAs were calculated using only categorized changes in either p300 or Srf to predict changes in H3ac (Fig. 5A and B). Both models indicated a significant dependency of $\mathrm{H} 3 \mathrm{ac}$ on the individual factor with an empirical $p$-value $<5 \times 10^{-5}$. The estimated coefficients for up and down regulated regions were significantly higher and lower than zero. The distribution of the residuals and the 
A

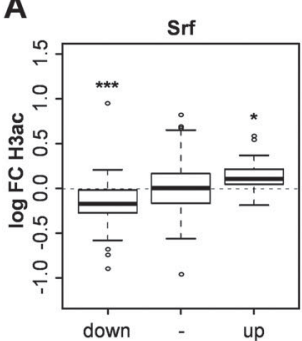

B

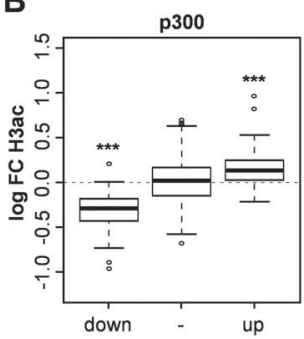

C

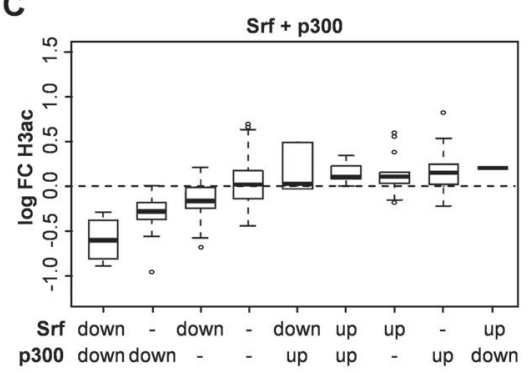

E

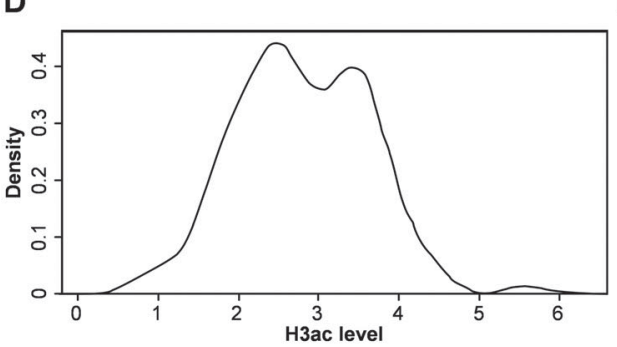

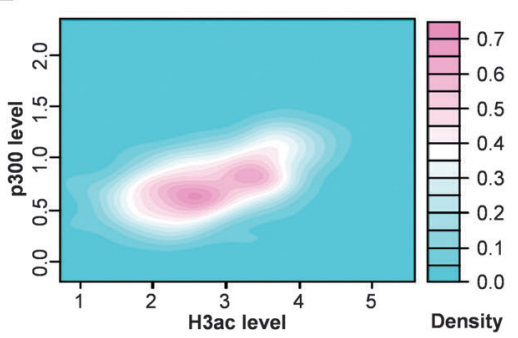

Fig. 5 (A-C) ANOVA models predicting changes in H3ac (combined time points). Boxplots illustrating the dependence of changes in H3ac from grouped significant changes in (A) Srf, (B) p300 and (C) Srf and p300. Significance levels indicating difference from zero according to the singlefactor ANOVA models are given above each box, using the following coding: ${ }^{*} p<0.05,{ }^{* * *} p<0.001$. Estimated coefficients and respective $p$-values for the two-factor ANOVA model can be found in Table 1. (D) Density of H3ac enrichment levels of region without a significant change in both Srf and p300 using a bandwidth of 0.253 and $n=227$ observations. (E) Two-dimensional density for H3ac and p300 enrichment levels. The height is indicated using the color key on the right.

normal Q-Q plots indicated validity of the linear model assumptions for both single-factor ANOVAs (Fig. S5, ESI $\dagger$ ).

After proving the dependency of $\mathrm{H} 3 \mathrm{ac}$ changes on each Srf and p300 individually, we were interested in the interaction between the two and if the observed changes were mainly dependent on the interplay between the two factors or on both factors independently. Therefore, a two-factor ANOVA was performed using categorized changes in both Srf as well as p300 as predictors for changes in H3ac (Fig. 5C). Again, the model indicated significant dependency with an empirical $p$-value $<5 \times 10^{-5}$. Like in the single-factor ANOVA, the estimated coefficients for the influence of up and down regulation of Srf and p300, which can be found in Table 1, were significantly different from zero, with the exception of up-regulated Srf regions. As a result, changes in both p300 and Srf had a significant influence on H3ac. Reduction of Srf binding on top of reduced p300 levels leads to further diminishment of $\mathrm{H} 3 \mathrm{ac}$, which substantiates the assumption that Srf triggers $\mathrm{H} 3 \mathrm{ac}$ additionally by a p300 independent mechanism. This effect appears not to be as pronounced for regions of elevated Srf and p300 levels. One possible explanation is that both p300 and Srf serve as necessary components for histone acetylation and thus reducing the level of each factor reduces H3ac. Increasing their levels may not increase $\mathrm{H} 3 \mathrm{ac}$ due to shortage of other necessary components. One suggestive co-player is Myocardin. Another possible explanation is the much lower number of regions with elevated Srf and p300 levels, which is likely correlated to the high number of genes that are active during development but are shut down afterwards. This lower number might have resulted in reduced statistical power for regions of elevated Srf and p300 levels. Like for the singlefactor ANOVAs, the distribution of the residuals and the normal Q-Q plots indicated validity of the linear model assumptions (Fig. S6, ESI $\dagger$ ).

To gain insights of $\mathrm{H} 3 \mathrm{ac}$ levels in regions with steady state levels of Srf and p300, we used density plots of mean levels for H3ac, p300 and Srf over all regions. We were interested if these stable Srf and p300 regions had a general preference in their absolute H3ac levels, such that higher p300 levels are associated with higher H3ac levels. Fig. 5D shows the density plot of mean levels for $\mathrm{H} 3 \mathrm{ac}$ over all regions. It indicates a bimodal distribution with two peaks that correlate with regions of high and low acetylation levels. The same two peaks were found in a two-dimensional density plot for absolute $\mathrm{H} 3 \mathrm{ac}$ and p300 levels (Fig. 5E). We did not observe a bimodal distribution for Srf (data not shown). However, in both plots the two peaks are not perfectly separated, indicating many regions with intermediate $\mathrm{H} 3 \mathrm{ac}$ and p300 levels.

In line with this qualitative assessment of $\mathrm{H} 3 \mathrm{ac}$ in steady state regions of $\mathrm{Srf}$ and $\mathrm{p} 300$, we were interested if the dynamics of H3ac levels were also quantitatively dependent on the dynamics of Srf and p300 levels. In other words, if stronger changes in any of

Table 1 Estimates and $p$-values of two-factor ANOVA. The $p$-values are based on $F$ statistic and reflect the significance of the estimates difference from zero. All estimates are in log space

\begin{tabular}{llllll}
\hline & - & p300 up & p300 down & Srf up & Srf down \\
\hline Coefficient & 0.03483 & 0.12557 & -0.32705 & 0.06896 & -0.18069 \\
$p$-value & $2.23 \times 10^{-2}$ & $1.9 \times 10^{-3}$ & $4.56 \times 10^{-14}$ & $1.84 \times 10^{-1}$ & $1.42 \times 10^{-4}$ \\
\hline
\end{tabular}


Table 2 Estimates and $p$-values of a linear regression model. The $p$-values are based on $F$ statistic and reflect the significance of the estimates difference from zero. All estimates are in log space

\begin{tabular}{llll}
\hline & Intercept & Srf & $\mathrm{p} 300$ \\
\hline Coefficient & 0.00622 & 0.31083 & 0.32681 \\
$p$-value & $5.68 \times 10^{-1}$ & $5.25 \times 10^{-12}$ & $1.12 \times 10^{-23}$ \\
\hline
\end{tabular}

these two factors lead to stronger changes in $\mathrm{H} 3 \mathrm{ac}$ and which of the two factors is more influential. Therefore, a linear regression model was computed predicting the strength of $\mathrm{H} 3 \mathrm{ac}$ fold changes from Srf and p300 fold changes directly. Like for the previous models, an empirical $p$-value was derived from random experiments. Again, the residuals and the Q-Q plots revealed validity of the linear model assumptions (Fig. S7A and B, ESI $\dagger$ ).

The linear regression model revealed that changes in $\mathrm{H} 3 \mathrm{ac}$ level were not only qualitatively but also quantitatively dependent on changes in Srf as well as p300 level $\left(p<5 \times 10^{-5}\right)$. In line with the results from the qualitative model, both factors showed a significant influence and to a roughly equal extend according to the high similarity in their estimated coefficients (Table 2). This finding further questions the proposed dependency between the two factors in the analyzed time-series, indicating p300-independent mechanisms of Srf-triggered H3ac. Finally, we computed the Pearson correlation coefficient between predicted and observed changes reaching an overall model accuracy of 0.67 (Fig. S7C, ESI $\dagger$ ). While far from perfect, this correlation demonstrates that changes in the two factors Srf and p300 already drive an appreciable fraction of the measured changes in histone 3 acetylation levels.

So far we used changes in $\mathrm{H} 3 \mathrm{ac}$ level as the predicted readout of changes in Srf and p300 level. In a last step, we were interested whether the observed changes also had a functional consequence on gene expression. Therefore, gene expression levels for 44 genes associated to one or multiple analyzed regulatory regions were measured in the same three stages using quantitative real-time PCR. Like for the ChIP measurements, a normalization step was introduced to remove potential trends in the mean expression over all measured genes between the three individual stages and fold changes were calculated to measure the effective change between the individual stages. To check whether changes in the individual factors lead to changes in gene expression, genes were grouped according to significant up or down regulated regions for each factor combining the two time points. Using a $t$-test between the two groups, we observed significant dependencies for changes of gene expression to changes of H3ac and Srf levels ( $p=$ 0.017 and $p=0.03$, respectively, Fig. 6A and B) and only an insignificant trend for p300 levels (data not shown).

In addition, we analyzed the dependency of gene expression changes on all measured factors and histone modifications. While the genes in general showed the proposed dependency on binding or modification changes, several were regulated by only subgroups of these (representative examples are shown in Fig. 6C-H). This finding likely indicates a high variability of combinatorial regulation between the investigated regulators. Though, it has to be kept in mind that we measured only three consecutive timepoints and might therefore have missed intermediate regulatory events.

\section{Summary and conclusion}

We investigated the interdependency between Srf and H3ac in conjunction with $\mathrm{H} 3 \mathrm{~K} 4 \mathrm{me} 2$ and the acetyltransferase p300 in vivo using a time-series of maturating murine cardiomyocytes around birth. With respect to the limited amount of cardiac material, regulatory regions were selected that showed binding of at least two of the three factors Srf, H3ac and $\mathrm{H} 3 \mathrm{~K} 4 \mathrm{me} 3$ in a preceding HL-1 study. These regions were subsequently profiled for their enrichment with the analyzed factors and histone modifications. Investigating changes between the successive stages, binding of the individual factors was in general found to be very dynamic with many significant changes between the individual stages. Most importantly, these changes showed high levels of correlation pointing to strong interdependencies. While a high correlation between changes of the histone acetyltransferase p300 and acetylation level was expected, the link between the other factors is not straightforward. Interestingly, the highest correlation was found between changes in $\mathrm{H} 3 \mathrm{ac}$ and $\mathrm{H} 3 \mathrm{~K} 4 \mathrm{me} 2$ levels. This supports findings from Wang et $a^{23}$ in human $\mathrm{CD} 4+\mathrm{T}$ cells which revealed that histones in promoters that showed initial $\mathrm{H} 3 \mathrm{~K} 4$ methylation were acetylated after treatment with Trichostatin A, an histone deacetyltransferase inhibitor, while those promoters that did not had initial $\mathrm{H} 3 \mathrm{~K} 4$ methylation remained largely unacetylated. The coupling between histone tail methylation and acetylation might either be achieved through chromatin remodelers binding both modifications such as Dpf3b (Baf45c $)^{28}$ or BPTF, ${ }^{29,30}$ the recruitment of ATP-dependent chromatin remodeling complexes which open the chromatin to allow other histone modification enzymes to bind ${ }^{24,25}$ or by direct $\mathrm{H} 3 \mathrm{~K} 4 \mathrm{me} 2$ recognition from histone acetylation complexes. ${ }^{26}$

Further, it has been suggested that $\mathrm{H} 3 \mathrm{~K} 4$ methylation increases the efficiency of p300 function and consecutive histone acetylation. ${ }^{31}$ In line with this we found significant correlation between changes in $\mathrm{H} 3 \mathrm{~K} 4 \mathrm{me} 2$ and p300 levels and finally coupling to $\mathrm{H} 3 \mathrm{ac}$, which might likely be established through tissue specific transcription factors. Following a model suggested by McDonald and Owens, ${ }^{13}$ gene activation by $\mathrm{Srf}$ is performed in a step-wise procedure. In order to bind its CArG-box motif, initial histone modifications like H3K4me2 and H4ac must be present in smooth muscle cells. $\mathrm{H} 4 \mathrm{ac}$ thereby leads to an open chromatin state facilitating genomic access while $\mathrm{H} 3 \mathrm{~K} 4 \mathrm{me} 2$ provides a docking site to the Srf/Myocardin complex. This complex has been suggested to recruit further transcription factors and transcriptional co-factors including p300 that fully activate the gene expression program. Here, we focused on cardiomyocytes maturation using an in vivo time-series model and suggest that $\mathrm{H} 3 \mathrm{ac}$ instead of H4ac plays a crucial role for Srf-driven gene expression. Both quantitative and qualitative modeling of changes in H3ac showed a significant dependency on Srf and p300 levels either alone or in conjunction. A key question was whether the implemented models would substantiate the stepwise regulatory model in which $\mathrm{H} 3 \mathrm{ac}$ levels are dependent on Srf binding through recruitment of p300 or if the presence of both factors independently influences H3ac levels. We found a minor correlation between Srf and p300 levels, however, both qualitative as well as quantitative linear modeling revealed a 

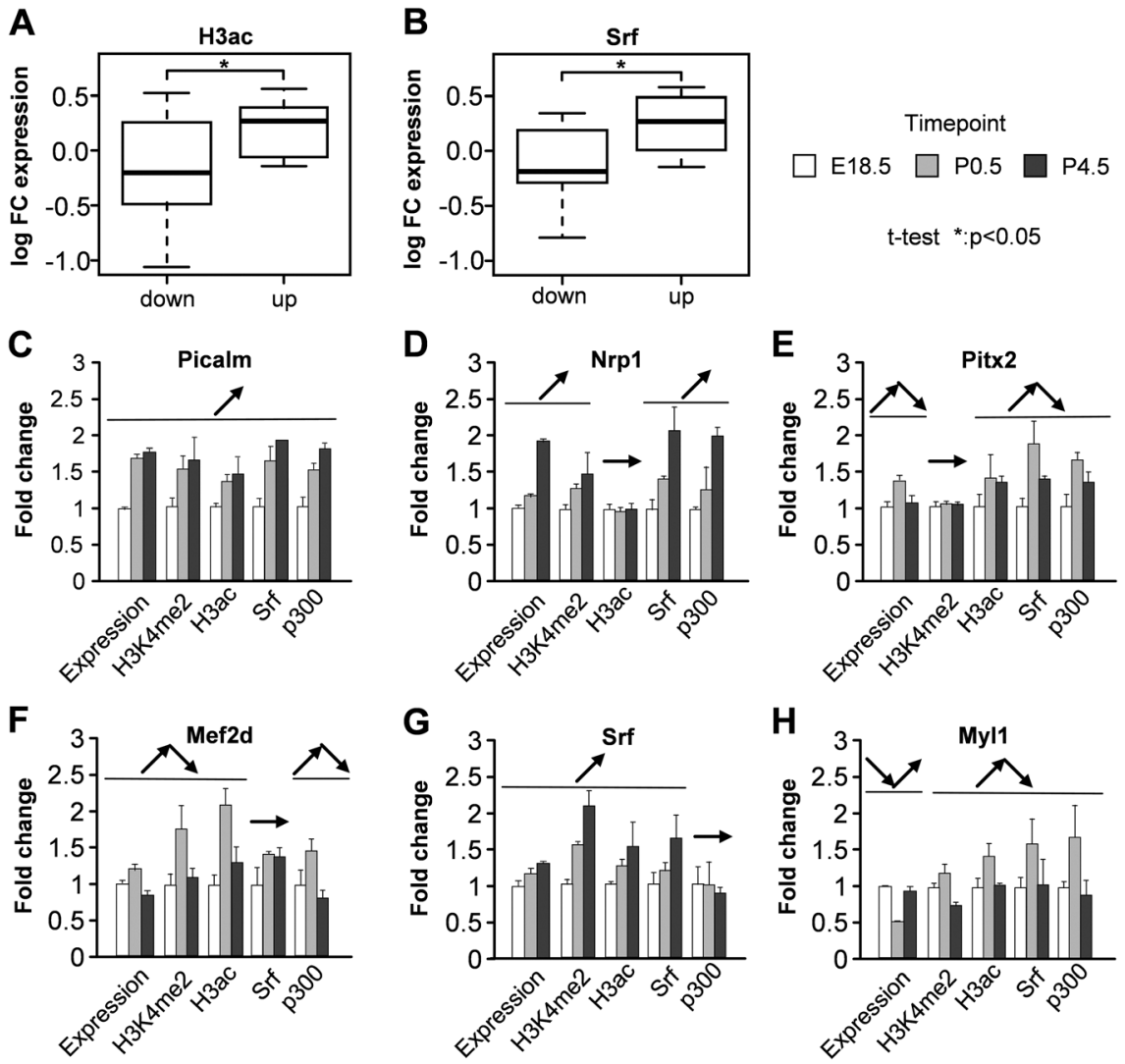

Fig. $6(\mathrm{~A}+\mathrm{B})$ Gene expression changes grouped by significant up or down regulation of H3ac and Srf. Boxplots illustrating the dependence of changes in expression level from significant changes in (A) H3ac and (B) Srf. (C-H) Representative examples of variable gene regulation. (C) All factors and histone modifications correlate with gene expression. (D-G) All factors and histone modifications correlate with gene expression with the exception of (D) H3ac, (E) H3K4me2, (F) Srf and (G) p300. (H) Anti-correlation between Srf, p300, histone modifications and gene expression.

significant influence of $\mathrm{Srf}$ as well as p300 on H3ac levels, pointing to independent regulation. We therefore strongly believe that Srf-associated H3ac is not only achieved via the recruitment of p300 but is established by the interaction with additional histone acetylation factors. Furthermore, we found for cardiac relevant genes that gene expression levels and the presence of H3ac and Srf binding are highly correlated which does not apply for p300. Notably, on the single-gene level a panel of regulatory dependencies can be observed with dynamic subsets of regulators that co-operatively work to regulate correct temporal transcription of target genes.

\section{Materials and methods}

\section{Cardiac samples}

Mouse hearts at the indicated stages of CD1 strain were dissected in cold PBS from the rest of the body. For subsequent RNA isolation heart samples were directly snap frozen in liquid nitrogen and stored at $-80{ }^{\circ} \mathrm{C}$. For ChIP experiments heart samples were minced and fixed with $1 \%$ formaldehyde solution.

\section{Chromatin immunoprecipitation followed by qPCR}

Chromatin immunoprecipitation (ChIP) experiments were performed using $30 \mathrm{mg}$ mouse heart tissue each. Tissue samples were minced and cross-linked for $15 \mathrm{~min}$ at room temperature with fixation buffer ( $1 \%$ formaldehyde, $10 \mathrm{mM} \mathrm{NaCl}, 100 \mu \mathrm{M}$
EDTA, $50 \mu \mathrm{M}$ EGTA, 5 mM HEPES, pH 7.5). Cross-linking was terminated by addition of glycine to a final concentration of $125 \mathrm{mM}$. Tissue samples were homogenized for disaggregation. Subsequently, cells were incubated for $15 \mathrm{~min}$ at $4{ }^{\circ} \mathrm{C}$ with lysis buffer (50 mM HEPES, pH 7.5, $140 \mathrm{mM} \mathrm{NaCl}$, $1 \mathrm{mM}$ EDTA, $1 \%$ Triton, $0.1 \%$ Na-deoxycholate, $0.1 \%$ SDS, $1 \mathrm{mM}$ PMSF, $1 \times$ Complete Protease Inhibitor Cocktail (Roche)), collected by centrifugation and homogenized using a Douncer. Nuclei were collected by centrifugation for $15 \mathrm{~min}$ at $20000 \mathrm{~g}$ and $4{ }^{\circ} \mathrm{C}$ and resuspended in sonification buffer (10 mM Tris- $\mathrm{HCl}, \mathrm{pH} 8.0,1 \mathrm{mM}$ EDTA and $0.5 \mathrm{mM}$ EGTA, $1 \mathrm{mM}$ PMSF, $1 \times$ Complete Protease Inhibitor Cocktail). The chromatin was fragmented by sonification to an average size of $500 \mathrm{bp}$. For immunoprecipitation buffer conditions were adjusted to the following concentrations: $140 \mathrm{mM} \mathrm{NaCl}, 1 \%$ Triton, $0.1 \%$ SDS, $0.1 \% \mathrm{Na}$-deoxycholate. A fraction of material was saved as "Input". Chromatin was pre-cleared by rotation with Dynabeads Protein A/G (Invitrogen) for $1 \mathrm{~h}$ at $4{ }^{\circ} \mathrm{C}$. Antibodies were added as given in Table $\mathrm{S} 1$ (ESI $\dagger$ ) and incubated on a rotating wheel over night at $4{ }^{\circ} \mathrm{C}$. Protein $\mathrm{A} / \mathrm{G}$ beads were added and rotation continued for $1 \mathrm{~h}$. The beads were washed twice with RIPA buffer A $(10 \mathrm{mM}$ Tris- $\mathrm{HCl}$, $\mathrm{pH} 8.0,140 \mathrm{mM} \mathrm{NaCl}, 0.025 \% \mathrm{NaN}_{3}, 1 \%$ Triton, $0.1 \% \mathrm{SDS}$, $1 \% \mathrm{Na}$-deoxycholate), once with RIPA buffer B (10 mM Tris- $\mathrm{HCl}, \mathrm{pH} 8.0,500 \mathrm{mM} \mathrm{NaCl}, 0.025 \% \mathrm{NaN}_{3}, 1 \%$ Triton, $0.1 \% \mathrm{SDS}, 1 \% \mathrm{Na}$-deoxycholate), once with $\mathrm{LiCl}$ detergent 
solution (10 mM Tris- $\mathrm{HCl}, \mathrm{pH} 8.0,0.5 \% \mathrm{Na}$-deoxycholate, $1 \mathrm{mM}$ EDTA, $250 \mathrm{mM} \mathrm{LiCl}, 0.5 \%$ Nonidet P-40) and once with TBS (20 mM Tris-HCl, pH 7.6, $150 \mathrm{mM} \mathrm{NaCl}$ ). Immunocomplexes were disrupted by eluting $10 \mathrm{~min}$ at $65{ }^{\circ} \mathrm{C}$ with $1 \% \mathrm{SDS} / \mathrm{TE}$ buffer $(10 \mathrm{mM}$ Tris-HCl, $\mathrm{pH}$ 7.6, $1 \mathrm{mM}$ EDTA, $1 \%$ SDS) and eluting a second time for 15 min with $0.67 \%$ SDS/TE buffer (10 mM Tris-HCl, pH 7.6, 1 mM EDTA, $0.67 \%$ SDS). Eluates were combined and reverse cross-linked by heating at $65^{\circ} \mathrm{C}$ over night. Subsequently, DNA was treated with RNase A and Proteinase $\mathrm{K}$ and purified by phenolchloroform extraction.

ChIP samples of Srf, p300, H3ac and H3K4me2 for 190 regions (Table S2, ESI $\dagger$ ) were analyzed with TaqMan qPCR in context of a beta-side testing of the LightCycler ${ }^{\mathbb{R}} 1536$ System from Roche Applied Science. TaqMan qPCR assays were designed using the Universal ProbeLibrary (UPL) and the ProbeFinder software which is freely accessible at www.roche-appliedscience.com. For the 1536-well plate format the Innovadyne ${ }^{\mathrm{TM}}$ Nanodrop $^{\mathrm{TM}}$ Express pipetting robot (IDEX Health \& Science LLC, Rohnert Park, CA, USA) and the Velocityl1 sealing machine (Agilent, Santa Clara, CA, USA) were used. ChIP analysis was carried out in triplicates using the following program: enzyme activation: $95^{\circ} \mathrm{C}$ for $1 \mathrm{~min}$; amplification ( 45 cycles): $95^{\circ} \mathrm{C}$ for $1 \mathrm{~s} \mathrm{(ramp:} 4.8^{\circ} \mathrm{C} \mathrm{s}^{-1}$ ), $60{ }^{\circ} \mathrm{C}$ for $30 \mathrm{~s}$ (ramp: $2.5^{\circ} \mathrm{C} \mathrm{s}^{-1}$ ); cooling: $40^{\circ} \mathrm{C}$ for $30 \mathrm{~s}$ (ramp: $2.5^{\circ} \mathrm{C} \mathrm{s}^{-1}$ ). The detection format was set to "Mono Color Hydrolysis/UPL Probes' and the pipetting control to 'Master Control'. The qPCR reaction contained $0.8 \mu \mathrm{l} \mathrm{ChIP} \mathrm{sample}$ and $1.2 \mu \mathrm{l}$ mastermix containing $1 \times$ RealTime ready DNA Probes Master, $300 \mathrm{nM}$ primer and $400 \mathrm{nM}$ probe.

\section{Preparation of protein extracts and Western Blot analysis}

Protein extraction and Western Blot were performed according to standard protocols. All antibodies with their respective dilution are given in Table S1 (ESI $\dagger$ ). Signals on the blots were subjected to densitometry measurements using NIH's ImageJ software.

\section{mRNA expression analysis}

Total RNA of heart tissues was isolated using TRIzol reagent (Invitrogen) followed by DNase digest (Promega) and ethanol precipitation according to standard protocols. Reverse transcription reactions were carried out via AMV-RT (Promega) with random hexamers (Amersham Pharmacia Biotech). To verify transcript expression levels of mouse hearts, quantitative real-time PCR measurements were performed using Absolute qPCR SYBR Green ROX Mix (Thermo Scientific) and the ABI PRISM 7900HT Sequence Detection System according to manufacturer's protocol. Gene expression was calculated using the $\Delta \mathrm{CT}$ method with normalization to the housekeeping gene Hprt. Genes and corresponding primer sequences can be found in Table S3 (ESI $\dagger$ ).

\section{Bioinformatic analysis}

All computations, linear models, statistical tests and plots were carried out using $\mathrm{R}^{32}$ The two-dimensional density plot was computed using the $k d e 2 d$ function from R's MASS package. ${ }^{33}$ Gene annotations were based on Ensembl, version 55.
To assess the influence of the individual factors on gene expression for genes with multiple assigned ChIP regions, the changes for these regions were combined using the following algorithm for each factor and time point comparison individually: At first, changes were categorized into up, down and unchanged as described in the manuscript. In a second step, genes that were associated to both up and down regulated regions were discarded from the analysis. Finally, genes which were associated to at least one region with a significant change were categorized accordingly and genes associated to only unchanged regions were discarded.

\section{References}

1 C. Simone, J. Cell. Physiol., 2006, 207, 309-314.

2 J. M. Miano, J. Mol. Cell. Cardiol., 2003, 35, 577-593.

3 O. G. McDonald, B. R. Wamhoff, M. H. Hoofnagle and G. K. Owens, J. Clin. Invest., 2006, 116, 36-48.

4 Q. Sun, G. Chen, J. W. Streb, X. Long, Y. Yang, C. J. Stoeckert, Jr. and J. M. Miano, Genome Res., 2006, 16, 197-207.

5 G. Schratt, U. Philippar, J. Berger, H. Schwarz, O. Heidenreich and A. Nordheim, J. Cell Biol., 2002, 156, 737-750.

6 R. O. Balza, Jr. and R. P. Misra, J. Biol. Chem., 2006, 281, 6498-6510.

7 S. Arsenian, B. Weinhold, M. Oelgeschlager, U. Ruther and A. Nordheim, EMBO J., 1998, 17, 6289-6299.

8 J. M. Miano, N. Ramanan, M. A. Georger, K. L. de Mesy Bentley, R. L. Emerson, R. O. Balza, Jr., Q. Xiao, H. Weiler, D. D. Ginty and R. P. Misra, Proc. Natl. Acad. Sci. U. S. A., 2004, 101, 17132-17137.

9 S. Li, M. P. Czubryt, J. McAnally, R. Bassel-Duby, J. A. Richardson, F. F. Wiebel, A. Nordheim and E. N. Olson, Proc. Natl. Acad. Sci. U. S. A., 2005, 102, 1082-1087.

10 D. Cao, Z. Wang, C. L. Zhang, J. Oh, W. Xing, S. Li, J. A. Richardson, D. Z. Wang and E. N. Olson, Mol. Cell. Biol., 2005, 25, 364-376.

11 I. Manabe and G. K. Owens, Circ. Res., 2001, 88, 1127-1134.

12 P. Qiu and L. Li, Circ. Res., 2002, 90, 858-865.

13 O. G. McDonald and G. K. Owens, Circ. Res., 2007, 100, $1428-1441$.

14 D. Wang, P. S. Chang, Z. Wang, L. Sutherland, J. A. Richardson, E. Small, P. A. Krieg and E. N. Olson, Cell, 2001, 105, 851-862.

15 N. D. Heintzman, R. K. Stuart, G. Hon, Y. Fu, C. W. Ching, R. D. Hawkins, L. O. Barrera, S. Van Calcar, C. Qu, K. A. Ching, W. Wang, Z. Weng, R. D. Green, G. E. Crawford and B. Ren, Nat. Genet., 2007, 39, 311-318.

16 A. Visel, M. J. Blow, Z. Li, T. Zhang, J. A. Akiyama, A. Holt, I. Plajzer-Frick, M. Shoukry, C. Wright, F. Chen, V. Afzal, B. Ren, E. M. Rubin and L. A. Pennacchio, Nature, 2009, 457, $854-858$.

17 J. Schlesinger, M. Schueler, M. Grunert, J. J. Fischer, Q. Zhang, T. Krueger, M. Lange, M. Tonjes, I. Dunkel and S. R. Sperling, PLoS Genet., 2010, 7, e1001313.

18 J. J. Fischer, J. Toedling, T. Krueger, M. Schueler, W. Huber and S. Sperling, Genomics, 2008, 91, 41-51.

19 A. Hirschy, F. Schatzmann, E. Ehler and J. C. Perriard, Dev. Biol., 2006, 289, 430-441.

20 F. J. Davis, M. Gupta, S. M. Pogwizd, E. Bacha, V. Jeevanandam and M. P. Gupta, Am. J. Physiol., 2002, 282, H1521-H1533.

21 P. R. Kemp and J. C. Metcalfe, Biochem. J., 2000, 345(Pt 3), 445-451.

22 C. M. Koch, R. M. Andrews, P. Flicek, S. C. Dillon, U. Karaoz, G. K. Clelland, S. Wilcox, D. M. Beare, J. C. Fowler, P. Couttet, K. D. James, G. C. Lefebvre, A. W. Bruce, O. M. Dovey, P. D. Ellis, P. Dhami, C. F. Langford, Z. Weng, E. Birney, N. P. Carter, D. Vetrie and I. Dunham, Genome Res., 2007, 17, 691-707.

23 Z. Wang, C. Zang, K. Cui, D. E. Schones, A. Barski, W. Peng and K. Zhao, Cell, 2009, 138, 1019-1031.

24 M. G. Pray-Grant, J. A. Daniel, D. Schieltz, J. R. Yates, 3rd and P. A. Grant, Nature, 2005, 433, 434-438. 
25 J. Wysocka, T. Swigut, T. A. Milne, Y. Dou, X. Zhang, A. L. Burlingame, R. G. Roeder, A. H. Brivanlou and C. D. Allis, Cell, 2005, 121, 859-872.

26 D. G. Martin, D. E. Grimes, K. Baetz and L. Howe, Mol. Cell. Biol., 2006, 26, 3018-3028.

27 J. Faraway, Practical Regression and Anova using R, 2002.

28 M. Lange, B. Kaynak, U. B. Forster, M. Tonjes, J. J. Fischer, C. Grimm, J. Schlesinger, S. Just, I. Dunkel, T. Krueger, S. Mebus, H. Lehrach, R. Lurz, J. Gobom, W. Rottbauer, S. AbdelilahSeyfried and S. Sperling, Genes Dev., 2008, 22, 2370-2384.
29 H. Li, S. Ilin, W. Wang, E. M. Duncan, J. Wysocka, C. D. Allis and D. J. Patel, Nature, 2006, 442, 91-95.

30 A. J. Ruthenburg, H. Li, D. J. Patel and C. D. Allis, Nat. Rev., 2007, 8, 983-994.

31 H. Wang, R. Cao, L. Xia, H. Erdjument-Bromage, C. Borchers, P. Tempst and Y. Zhang, Mol. Cell, 2001, 8, 1207-1217.

32 R: A language and environment for statistical computing, 2005.

33 W. N. Venables, B. Ripley and W. N. Venables, Modern applied statistics with $S$, Springer, 2002. 